\title{
Progress in Computational Aeroacoustics in Predicting the Noise Radiated from Turbulent Flows
}

\author{
Geoffrey M. Lilley, Xin Zhang and Aldo Rona* \\ Department of Aeronautics and Astronautics, University of Southampton, SO17 1BJ, United Kingdom
}

\begin{abstract}
In recent years a number of simple unsteady flows involving the interaction between vortices have been studied using computational fluid dynamics. These have been extended to include the sound radiated to the far field either by Direct Numerical Simulation, by the use of acoustic analogies, or by the use of Kirchoff methods. For more complex flows results have been obtained using methods based on solving the time dependent large scale flow structures using the unsteady Reynolds Averaged Navier-Stokes equations and then using acoustic analogies to derive the noise in the radiation field. Some success has been made with the latter methods in the predictions of the noise radiated from the flow over cavities at supersonic speeds, where the noise characteristics are dominated by large scale events associated with self-excited flow oscillations. Similar methods are being applied to other self-excited flows, and ultimately to turbulent flows such as jets. The paper describes these methods and results together with some limited preliminary comparisons with experimental data. In an Appendix an extension of Lighthill's equation for aerodynamic noise is presented covering the effects of flow-acoustic interaction.
\end{abstract}

*Fourth International Congress on Sound and Vibration, St. Petersburg, Russia, June 24-27, 1996

\section{INTRODUCTION}

In recent years computational aeroacoustics has been shown to be a growing powerful tool in the prediction of the radiated noise from unsteady flows. At low Reynolds numbers Colonius et al. ${ }^{1}$ and Mitchell et al. ${ }^{2}$ have extended the method of Direct Numerical Simulation (DNS) for calculations of the radiated sound from shear layers. In these calculations it was found that one of the dominant noise sources involved the interaction of vortices and in particular their pairing. This was one of the noise sources Laufer had argued was a prime noise source in a jet and pioneered research on this subject throughout the 1970's and early 1980's (see Laufer and $\mathrm{Yen}^{3}$ ). The calculated unsteady flow results of Colonius et al. and Mitchell et al. were also used to predict the far field radiated noise using Lighthill's acoustic analogy ${ }^{4}$ and by using Kirchhoff ${ }^{\prime}$ s theorem. Good agreement was obtained except at shallow angles to the jet where the use of Lighthill's equation significantly overpredicted the far-field sound possibly due to the neglect of refraction of the sound by the mean flow. Even for these flows of relatively simple geometry the computations are expensive since, unlike standard CFD calculations, they must be time and space accurate. They are only possible when the number of modes and frequencies are strictly limited. Although such pioneering work can not handle fully developed turbulence it clearly is important in examining the methodology to be followed in the numerical study of aeroacoustics.

Further extensive calculations have been undertaken by Colonius et al. ${ }^{1}$ on the complex interaction between the sound generated and the flow field using both DNS and also making comparisons with the extension to Lighthill's equation to include flow-acoustic interaction as proposed by Lilley and Goldstein ${ }^{5}$. The DNS results were found to be in good agreement with solutions to the Goldstein-Lilley equation, and displayed the importance of refraction in flow fields having extensive volumes in the streamwise direction.

For turbulent flows the main attack has been made by Sarkar and Hussaini ${ }^{6}$ and Lilley ${ }^{7}$ on studying isotropic turbulent flows at low Mach numbers and low Reynolds numbers using the space-time flow data base to predict the radiated noise using Lighthill's acoustic analogy. As reported above, such low Reynolds number data is unrepresentative of fully developed turbulent flows. Although databanks exist for compressible isotropic turbulent flows, no results have so far been reported of the retarded-time space correlations required for the prediction of the far field radiated sound. An approximate extension of the incompressible space-time data has been reported by Lilley ${ }^{8}$ for the case of temperature fluctuations and the use of the isotropic turbulence model as an approximate predictor for the noise radiated from a jet over a wide range of Mach numbers and temperatures. What is urgently needed is the numerical calculation of the characteristics of the fourth-order space-time covariance as a function of typical homogeneous and non-homogeneous turbulent shear flows to validate the above results and establish if it is possible to use them as the building blocks for the prediction of noise in more complex flows. Such computations are, however, expensive since they require an accurate resolution in space and time. These statements in respect to DNS apply also to methods of Large Eddy Simulation (LES).

In a number of flows of practical importance it has been found that the flow is dominated by large scale structures and that the flow, including the large scale structures, can be calculated using the Reynolds Averaged Time Dependent Navier-Stokes equations. Once the unsteady flow has been solved to the necessary accuracy the radiated noise can then be calculated using Lighthill's acoustic analogy or if needs be, one of the more complex acoustic analogies when impor- 